\title{
Rotation rates of massive stars in the Magellanic Clouds
}

\author{
Laura R. Penny ${ }^{1}$ and Douglas R. Gies ${ }^{2}$ \\ ${ }^{1}$ Dept. of Physics \& Astronomy, College of Charleston, \\ Charleston, SC, USA \\ email: pennyl@cofc.edu \\ ${ }^{2}$ Dept. of Physics \& Astronomy, Georgia State University, \\ Atlanta, GA, USA \\ email: gies@phy-astr.gsu.edu
}

\begin{abstract}
We present the results of our survey of the projected rotational velocities of 161 O-type stars in the Magellanic Clouds from archival FUSE observations. The evolved and unevolved samples from each environment are compared through the Kolmogorov-Smirnov test to determine if the distribution of equatorial rotational velocities is metallicity dependent for these massive objects. Stellar interior models predict that massive stars with SMC metallicity will have significantly reduced angular momentum loss on the main sequence compared to their Galactic counterparts. Our statistical results find some support for this prediction but also show that even at Galactic metallicity, evolved and unevolved massive stars have fairly similar fractions of stars with large $\mathrm{V}$ sin i. What is more compelling are the few evolved objects in the Magellanic Clouds with rotational velocities that approach or even exceed those predicted from the evolutionary models.
\end{abstract}

Keywords. stars: rotation, stars: early-type, stars: evolution, ultraviolet: stars

\section{Introduction and Methodology}

Here we present the results of our project to test the treatment of angular momentum in the new stellar interior models through a large scale survey of projected rotational velocities of O-type stars in three metallicity environments: the Milky Way $\left(Z_{M W}=0.020\right)$, $\operatorname{LMC}\left(Z_{L M C}=0.007\right)$, and SMC $\left(Z_{S M C}=0.004\right)$. The Far Ultraviolet Spectrographic Explorer (FUSE) archive at the Multimission Archive at Space Telescope (MAST) contains spectra of $161 \mathrm{LMC}$ and SMC stars with spectral classes between B2 - O2. The targets are 120 evolved (luminosity classes I, II, \& III) and 41 unevolved (IV \& V luminosity classes) stars in these low $Z$ environments. These observations, along with 97 archival spectra of Galactic O-type stars, were obtained from MAST. Target star spectra are cross-correlated with that of a relatively narrow-lined template star. The CCF is the sum of the square of the differences between the test spectrum and the reference spectrum shifted in velocity from $-1000 \mathrm{~km} \mathrm{~s}^{-1}$ to $+1000 \mathrm{~km} \mathrm{~s}^{-1}$ at $10 \mathrm{~km} \mathrm{~s}^{-1}$ intervals. The functions are then rectified, inverted, and Gaussian fitted to obtain an estimate of the full-width at half-maximum (FWHM). Then using the calibrations developed from stars with known projected rotational velocities, a $V \sin i$ value is determined from the Gaussian width of each fitted CCF (for more details see Penny \& Gies 2009).

\section{Analysis}

We divide the data into the following samples: SMC unevolved (32 stars), SMC evolved (19 stars), LMC unevolved (36 stars), and LMC evolved (42 stars). We also create samples 


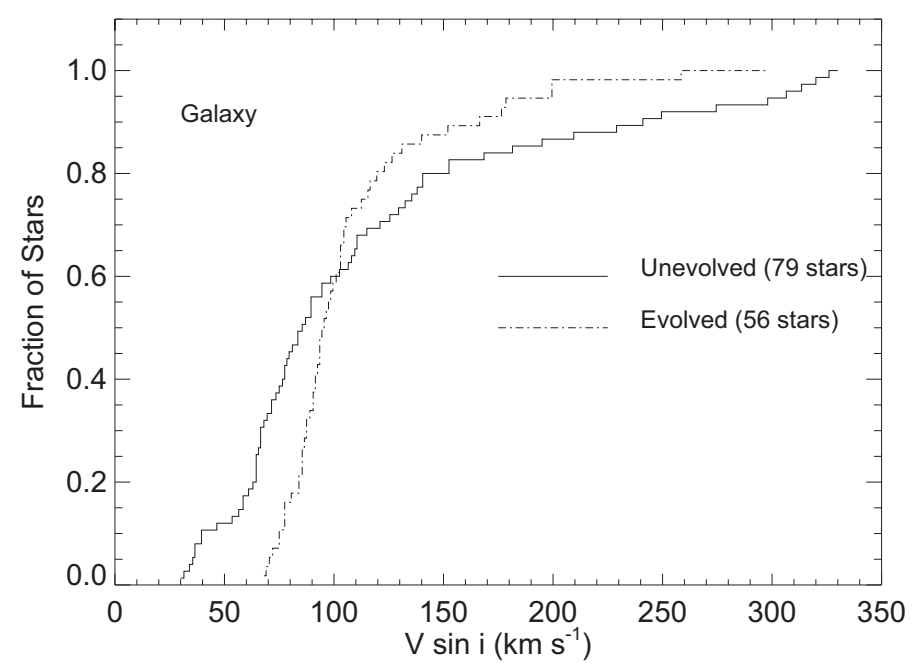

Figure 1. Cumulative distribution functions of $V \sin i$ values for unevolved and evolved stars in the Milky Way.

for Galactic unevolved (79 stars) and evolved (56 stars) from Howarth et al. (1997). Following the convention of earlier studies (Penny 1996, Howarth et al. 1997, Mokiem et al. 2006, Mokiem et al. 2007), unevolved refers to stars with luminosity classes V \& IV, while luminosity classes I \& II are termed evolved. Class III stars are omitted from both groups. For each sample we create a CDF and plot these in comparison with other samples to determine the similarity. For each comparison a K-S statistic, $D$, and its corresponding probability, $p$, are calculated and these are presented in Table 1 . Plots of the comparisons made are presented in Figures $1-6$.

First we examine the evolved and unevolved Galactic samples to determine the level of difference we would expect in an environment where the stars do slow down as they evolve (Fig. 1). We see that the maximum variance in their CDF is 0.30 and this occurs at $V \sin i$ of $83 \mathrm{~km} \mathrm{~s}^{-1}$. This large a $D$ value results in a $p$ of $0.5 \%$, but we should not be misled by this low value. At these smaller velocities the source of the difference between the evolved and unevolved samples is not a result of angular momentum loss, but from the larger amount of macroturbulence that is present in the evolved stars' photospheres, broadening their line profiles. At the higher velocities where we expect to see evidence of spin down, the largest divergence is $\approx 0.12$, which would give us a significantly higher $p$ of $71 \%$. This is not say that the evolved stars in the Galactic sample have not slowed down, but that this effect may be more subtle than we originally expected.

In the LMC, the same comparison of unevolved to evolved stars has a very different appearance (Fig. 2). The CDF of both samples appear very similar, and the derived $D=0.15$ and corresponding $p=75 \%$ supports the null hypothesis that both are drawn from the same parent distribution. This result is in agreement with the suggestion of Wolff et al. (2008), using data from Hunter et al. (2008), that massive stars in the LMC have similar $V \sin i$ distributions regardless of their $\log g$ values. Examining Fig. 2, we notice that there is almost no divergence at low $V \sin i$ values, and a smaller variance at larger $V \sin i$ than we see in the Milky Way samples. The very high $p$ value here is primarily due to the presence of slow rotators among both the unevolved and evolved samples, unlike the Galactic evolved group. At high $V \sin i$ values, the CDFs of evolved to unevolved differ by $\approx 0.09$ which is only slightly smaller than the value for the Galactic samples. It is at the low velocity end of the CDF where the LMC samples differ from 


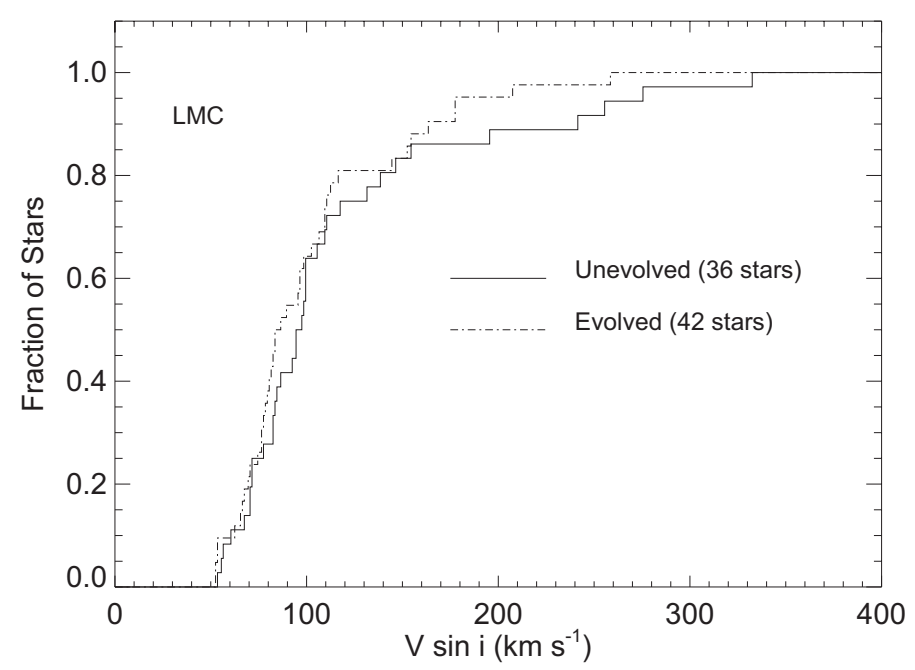

Figure 2. Cumulative distribution functions of $V \sin i$ values for unevolved and evolved stars in the LMC.

those of the Galactic samples. The good agreement between the low ends of the two CDFs for the LMC stars suggests that development of macroturbulence with evolution is not as large a factor in the photospheres of the massive stars in that environment and that the processes that lead to macroturbulent broadening may have a metallicity dependence.

The CDFs of the same populations in the SMC are presented in Figure 3. Although the $p$ value from the KS test is $23 \%$, which would indicate that the samples are drawn from the same parent population, the maximum difference $D$ is 0.29 , which is very close to that from the Galactic samples. The larger $p$ value from the similar $D$ reflects the much smaller sample sizes in the SMC. At the high velocity end of the CDF, the fraction of evolved stars with $V \sin i$ above $200 \mathrm{~km} \mathrm{~s}^{-1}$ is $11 \%$ compared to $13 \%$ for the unevolved. This does support the hypothesis that stars at SMC metallicity will not slow down as they evolve on the MS. At the other end of the CDF, there is a slightly larger fraction of unevolved stars with $V \sin i$ below $\approx 70 \mathrm{~km} \mathrm{~s}^{-1}$, but this difference is much smaller than we see in the Galactic comparison. The real divergence between the evolved and unevolved CDFs comes at the intervening velocities. The maximum divergence, 0.29, occurs at $V \sin i=107 \mathrm{~km} \mathrm{~s}^{-1}$. The fractions of stars with $V \sin i$ at or below this value for the evolved and unevolved samples are 0.84 and 0.55 , respectively. This trend was also seen by Mokiem et al. (2006), who surmised that the initial rotational velocity distribution in the SMC might vary significantly from that in the Galaxy. Why we see a disparity in the CDFs of the evolved and unevolved samples in between $80-190 \mathrm{~km} \mathrm{~s}^{-1}$ only in the SMC samples is not clear. We emphasize that the K-S test results accept the null hypothesis that the unevolved and evolved samples in the SMC are drawn from the same parent distribution.

A primary purpose of this project is to examine observationally the amount of angular momentum loss during the MS lifetimes of massive stars and the effects of metallicity on this loss. Comparing the evolved to unevolved samples in Figures $1-3$, we see that the $D$ statistic at high $V \sin i$ values range from $0.12,0.09,0.02$ in the Galaxy, LMC, and SMC, respectively. Taken by itself this is suggestive of a trend with decreasing $Z$. However, for the LMC and SMC comparisons, $D$ statistics of 0.09 and 0.02 result in $p$ values of $99.6 \%$ and $100 \%$, indicating there is no statistical difference between the evolved and 


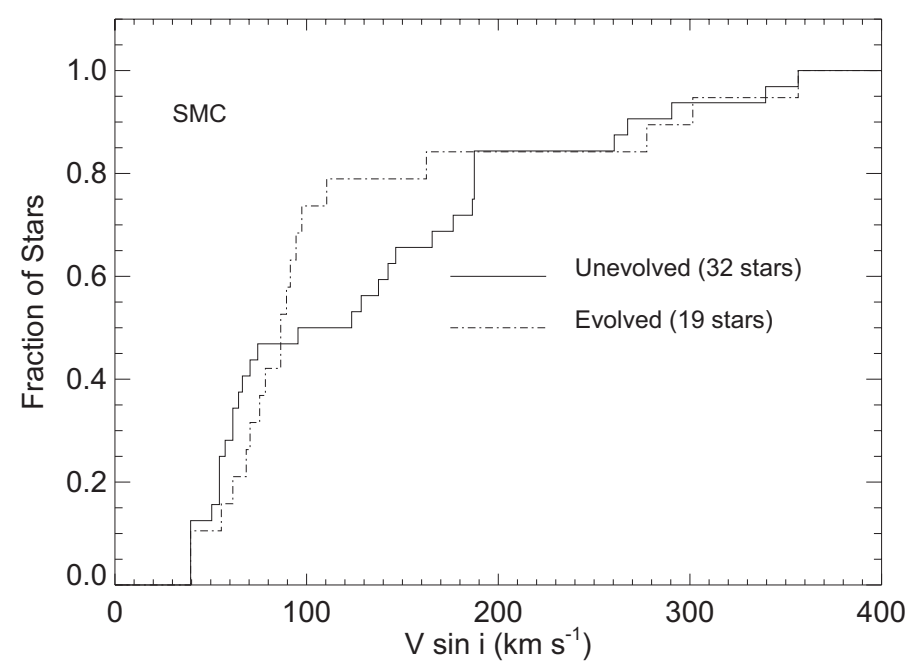

Figure 3. Cumulative distribution functions of $V \sin i$ values for unevolved and evolved stars in the SMC.

unevolved CDFs at high $V \sin i$. But this is also true in the Galaxy where we do expect to see the significant spin down between the evolved and unevolved samples. Here the corresponding $p$ for a $D$ of 0.12 is $71 \%$, far above the $5 \%$ cutoff. Statistically the loss of angular momentum for these H-burning stars is not so different between these three metallicity environments.

We also are interested in whether the initial $V \sin i$ distribution is the same in the three environments. In Figure 4, we plot the CDFs of all three dwarf samples. Statistically all three have $p$ values that are above the cut-off of $5 \%$, and certainly at the high $V \sin i$ end all three look extremely similar, indicating that the maximum rotational velocities are very similar. Below $200 \mathrm{~km} \mathrm{~s}^{-1}$, the behavior of the three distributions varies. Again we see that the shape of the CDF for the SMC dwarfs is dissimilar from that of the counterparts in the Galaxy and the LMC between $80-190 \mathrm{~km} \mathrm{~s}^{-1}$. Below $80 \mathrm{~km} \mathrm{~s}{ }^{-1}$, the fraction of slowly rotating stars varies between the three environments, with the Galactic sample in between the SMC and LMC. In fact the largest divergence between the LMC and SMC CDFs comes at $V \sin i \approx 65 \mathrm{~km} \mathrm{~s}^{-1}$, resulting in a $p$ value just above the statistically significant level. Hunter et al. (2008) showed an analogous plot, but for objects with $M<25 M_{\odot}$. Similarly they find good agreement between the LMC and Galactic samples, with the SMC sample lying slightly beneath the other two, especially in the region below $200 \mathrm{~km} \mathrm{~s}^{-1}$. It is interesting that there are no very slow rotators in our LMC dwarf sample. At these low velocities, the dominating effect must not depend upon metallicity since the Galaxy's metallicity, with significantly higher $Z$, is situated between that of the two low $Z$ samples. We conclude that the initial velocity distribution in our three unevolved samples are statistically indistinguishable.

Finally we compare the evolved stars in each environment to examine the relative fractions of stars with large $V \sin i$ (Fig. 5). Looking at the distributions at the high velocity end, we see that a slightly larger fraction of evolved stars in the SMC have $V \sin i$ values larger than $200 \mathrm{~km} \mathrm{~s}^{-1}$ than in the Galaxy or LMC, which might support theoretical predictions. But the large $D$ values that are found between the LMC and SMC samples are again at the low velocity range, $\approx 80 \mathrm{~km} \mathrm{~s}^{-1}$. The maximum differences between the Galaxy and both the LMC and SMC CDFs occur near this velocity, with both of the low metallicity environments having a larger fraction of stars with smaller 


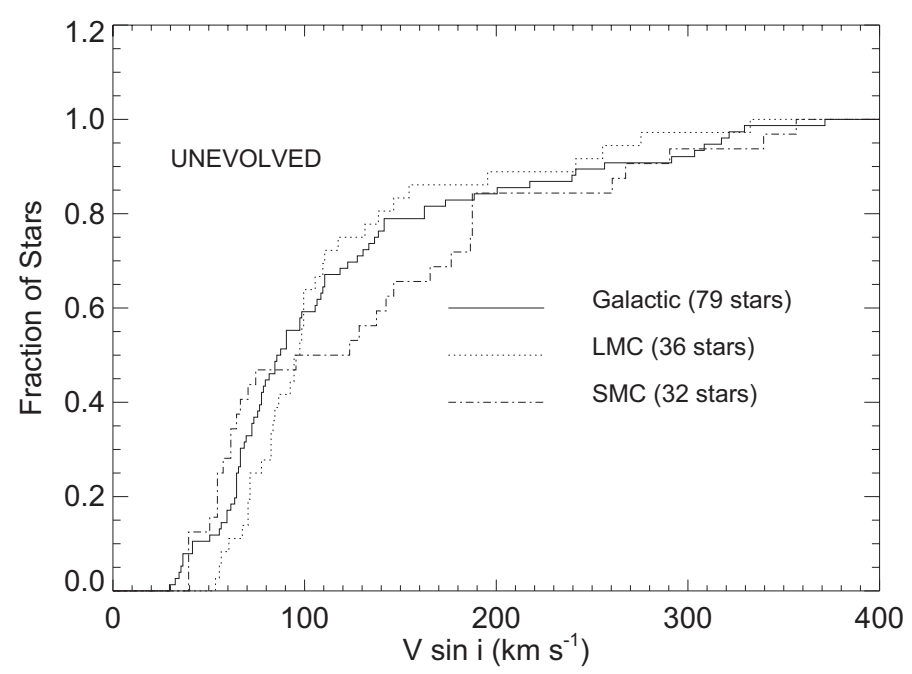

Figure 4. Cumulative distribution functions of $V \sin i$ values for unevolved (luminosity classes IV - V) stars in the SMC, LMC and Galaxy.

values compared to the Milky Way sample. In fact the trend is very supportive of our earlier suggestion that metallicity plays an important role in macroturbulent broadening in evolved O-type stars. A recent paper by Cantiello et al.(2009) discusses the origin of atmospheric turbulence in massive stars by sub-surface convection zones that are driven by Fe-peak element ionizations. In their simulations, the threshold luminosity for the occurrence of an iron convective zone is ten times lower at $Z_{M W}$ than that for $Z_{S M C}$. Our results support their prediction that turbulence will increase with metallicity. The SMC evolved sample has the largest fraction of slow rotators, followed by the LMC and then the Galaxy. We note that the $D$ values for the SMC vs. Galaxy and LMC vs. Galaxy are extremely similar, but result in differing $p$ values owing to the smaller SMC sample size. The large divergence at small $V \sin i$ values between the Galaxy and LMC, and possibly SMC, leads us to reject the hypothesis that they are both from the same parent distribution. Again we stress that the differences that cause this are not the fractions of stars with large $V \sin i$, but those with small values.

In conclusion, we find some support for the new stellar interior model predictions that massive stars in lower metallicity environments will remain at almost constant rotation rates throughout their MS lifetimes. But we also see that this effect, loss of angular momentum during the MS, is more subtle than previously reported even at the higher Galactic metallicity. We have suggested that metallicity may play an important role in the development of macroturbulence in the photospheres of the evolved, massive stars. Finally we note that there are several evolved stars in the SMC and LMC with very large $V \sin i$ values. Notable amongst these are: AV 321 (O9 Ib, $357 \mathrm{~km} \mathrm{~s}^{-1}$ ) and SK 190 (O8 Iaf, $302 \mathrm{~km} \mathrm{~s}^{-1}$ ).

\section{References}

Cantiello, M., Langer, N., Brott, I., de Koter, A. et al. 2009, Communications in Asteroseismology 158,61

Howarth, I. D., Siebert, K. W., Hussain, G. A. J., \& Prinja, R. K. 1997, MNRAS, 284, 265

Hunter, I., Lennon, D. J., Dufton, P. L., Trundle, C. et al. 2008, A $\& A, 479,541$

Mokiem, M. R., de Koter, A., Evans, C. J., Puls, J. et al. 2006, A\&A, 456, 1131 


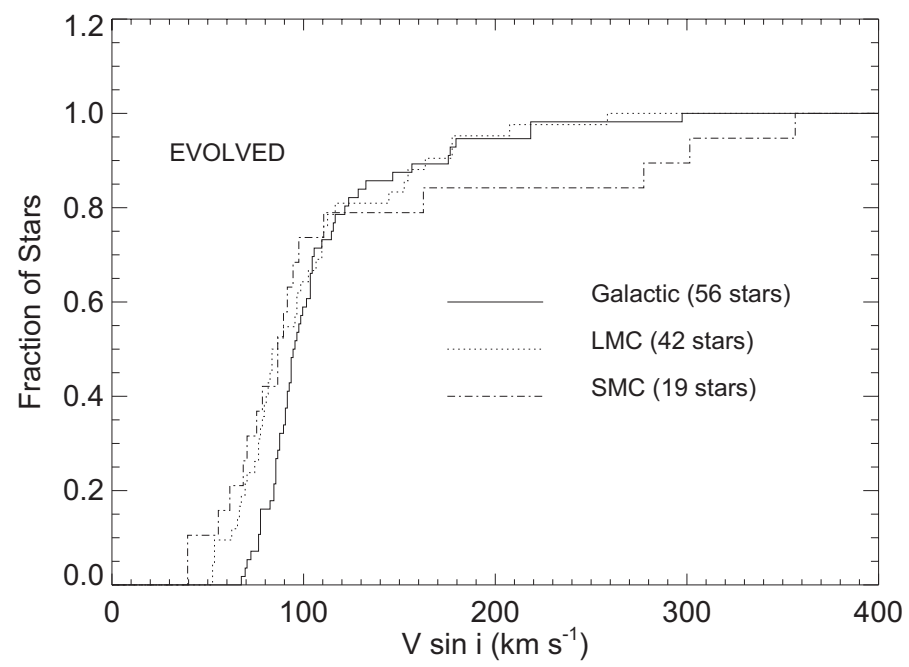

Figure 5. Same as Figure 4 but for evolved (luminosity classes I -II) stars in the same three environments.

Table 1. Kolmogorov-Smirnov Statistics

\begin{tabular}{|c|c|c|c|c|c|c|c|}
\hline Sample 1 & $\begin{array}{c}n_{1} \\
\text { stars }\end{array}$ & $\begin{array}{c}<V \sin i> \\
\left(\mathrm{km} \mathrm{s}^{-1}\right)\end{array}$ & Sample 2 & $\begin{array}{c}n_{2} \\
\text { stars }\end{array}$ & $\begin{array}{c}<V \sin i> \\
\left(\mathrm{km} \mathrm{s}^{-1}\right)\end{array}$ & $D$ & $\begin{array}{r}p \\
(\%)\end{array}$ \\
\hline \multicolumn{8}{|c|}{ Unevolved Vs. Evolved Within Each Environment } \\
\hline Galactic unevolved & 79 & 129.8 & Galactic evolved & 56 & 109.5 & 0.296 & 0.5 \\
\hline LMC unevolved & 36 & 132.0 & LMC evolved & 42 & 118.6 & 0.151 & 74 \\
\hline SMC unevolved & 32 & 116.2 & SMC evolved & 19 & 101.8 & 0.289 & 23 \\
\hline \multicolumn{8}{|c|}{ Unevolved Samples } \\
\hline LMC unevolved & 36 & 132.0 & Galactic unevolved & 79 & 129.8 & 0.180 & 37 \\
\hline SMC unevolved & 32 & 116.2 & Galactic unevolved & 79 & 129.8 & 0.172 & 47 \\
\hline SMC unevolved & 32 & 116.2 & LMC unevolved & 36 & 132.0 & 0.295 & 8 \\
\hline \multicolumn{8}{|c|}{ Evolved Samples } \\
\hline LMC evolved & 42 & 118.6 & Galactic evolved & 56 & 109.5 & 0.321 & 1 \\
\hline SMC evolved & 19 & 101.8 & Galactic evolved & 56 & 109.5 & 0.297 & 13 \\
\hline SMC evolved & 19 & 101.8 & LMC evolved & 42 & 118.6 & 0.137 & 95 \\
\hline
\end{tabular}

Mokiem, M. R., de Koter, A., Evans, C. J., Puls, J. et al. 2007, A\& A, 465, 1003

Penny, L. R. 1996, ApJ, 463, 737

Penny, L. R. \& Gies, D. R. 2009, ApJ, 700, 844

Penny, L. R., Sprague, A. J., Seago, G., \& Gies, D. R. 2004, ApJ, 617, 1316

Wolff, S. C., Strom, S. E., Cunha, K., Daflon, S. et al. 2008, AJ, 136, 1049 